\title{
Comprehensive prognostic report of the Japanese Breast Cancer Society Registry in 2004
}

\author{
Takayuki Kinoshita $^{1} \cdot$ Naohito Fukui ${ }^{2} \cdot$ Keisei Anan $^{3} \cdot$ Takayuki Iwamoto $^{4}$. \\ Naoki Niikura ${ }^{5}$ Masaaki Kawai ${ }^{6} \cdot$ Naoki Hayashi $^{7} \cdot$ Kouichiro Tsugawa $^{8}$. \\ Kenjiro Aogi ${ }^{9}$ Takanori Ishida ${ }^{10}$ - Hideji Masuoka ${ }^{11}$ - Shinobu Masuda ${ }^{12}$. \\ Kotaro Iijima $^{13} \cdot$ Seigo Nakamura ${ }^{14}$. Yutaka Tokuda ${ }^{5}$
}

Received: 20 May 2015 / Accepted: 27 July 2015/Published online: 7 October 2015

(C) The Author(s) 2015. This article is published with open access at Springerlink.com

Keywords Breast cancer - Prognosis - Report - Japan . Registry $\cdot 2004 \cdot$ The Japanese Breast Cancer Society

\section{Preface}

In 1975, the Breast Cancer Study Group (the predecessor of the Japanese Breast Cancer Society) initiated the Breast Cancer Registry and had registered 188,265 breast cancer patients during the 29 years from 1975 to 2003. In 2004, a new registration system was implemented, which had registered a total of 207,468 patients up to 2009 .

When the Personal Information Protection Law came into effect in 2004, the previous paper-based registration

Electronic supplementary material The online version of this article (doi:10.1007/s12282-015-0644-5) contains supplementary material, which is available to authorized users.

Yutaka Tokuda

tokuda@is.icc.u-tokai.ac.jp

1 Division of Breast Surgery, National Cancer Center Hospital, Tokyo, Japan

2 The Japan Clinical Research Support Unit, Tokyo, Japan

3 Department of Surgery, Kitakyushu Municipal Medical Center, Kitakyushu, Japan

4 Department of Breast and Endocrine Surgery, Okayama University Hospital, Okayama, Japan

5 Department of Breast and Endocrine Surgery, Tokai University School of Medicine, 143 Shimokasuya, Isehara, Kanagawa 259-1193, Japan

6 Department of Breast Surgery, Miyagi Cancer Center, Natori, Japan

7 Department of Breast Surgery, St. Luke's International Hospital, Tokyo, Japan system was converted to the web-based system, which includes anonymized efficacy data. At the same time, the data center was moved from the National Cancer Center to the Japan Clinical Research Support Unit (J-CRSU), a nonprofit organization, and the Public Health Research Foundation.

Herein, we are pleased to report, for the first time under the new system, results on five-year prognosis of patients that were registered in 2004 (Figs. 1, 2, 3, 4, 5, 6, 7, 8, 9; Supplementary Tables 1-9). We are deeply grateful to the medical and administrative staff as well as the patients who co-operatively participated in this study.

A total of 16,087 patients were originally registered from 317 institutions in 2004. Of these, 8585 (53.4\%) patients from 156 institutions were included in this prognostic study; we need to promote further participation in

8 Division of Breast and Endocrine Surgery, Department of Surgery, St. Marianna University School of Medicine, Kawasaki, Japan

9 Department of Breast Surgery, Shikoku Cancer Center, Matsuyama, Japan

10 Department of Surgical Oncology, Graduate School of Medicine, Tohoku University, Sendai, Japan

11 Sapporo-Kotoni Breast Clinic, Sapporo, Japan

12 Department of Pathology, Nihon University School of Medicine, Tokyo, Japan

13 Department of Breast Oncology, Cancer Institute Hospital, Tokyo, Japan

14 Division of Breast Surgical Oncology, Department of Surgery, Showa University, Tokyo, Japan 
prognostic studies in the future. We believe that continuing the study based on this registration system to assess the prognosis of breast cancer will contribute to improvements in breast cancer treatment in Japan and consequently the welfare of breast cancer patients, the ultimate goal of breast cancer care.
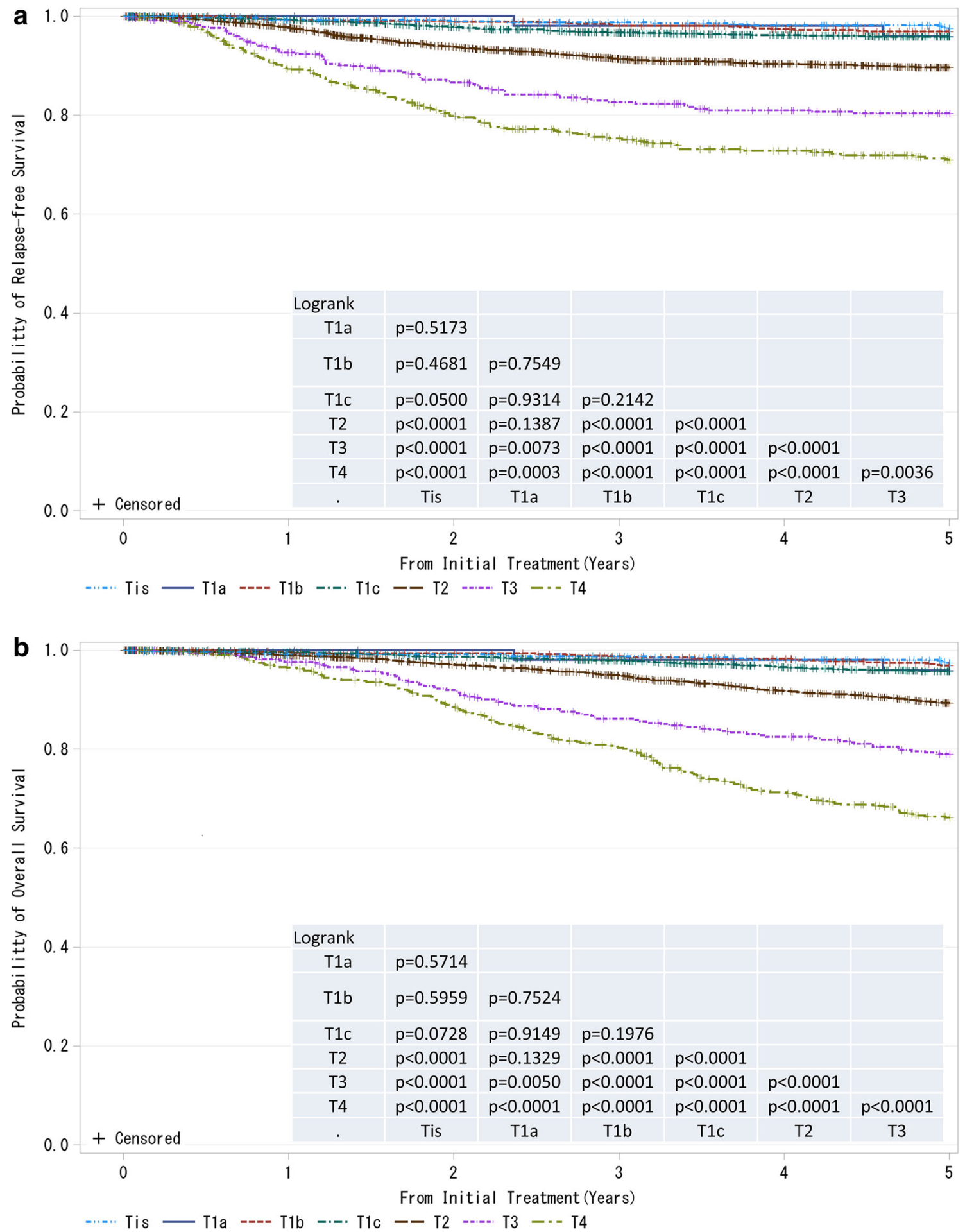

Fig. 1 a, b Kaplan-Meier curves for relapse-free and overall survival of all cases by tumor classification (cT-category). $P$ values were calculated using the log-rank test. Tis: Non-invasive ductal carcinoma, lobular carcinoma in situ, or Paget disease; T1a: $\leq 0.5 \mathrm{~cm}$;
T1b: $\quad 0.5<$ tumor $\leq 1.0 \mathrm{~cm} ; \quad$ T1c: $\quad 1.0<$ tumor $\leq 2.0 \mathrm{~cm}, \quad$ T2: $2.0<$ tumor $\leq 5.0 \mathrm{~cm} ; \mathrm{T} 3:>5.0 \mathrm{~cm}$; T4: tumor of any size with direct extension to the chest wall and/or skin (ulceration or skin nodules) or inflammatory carcinoma 

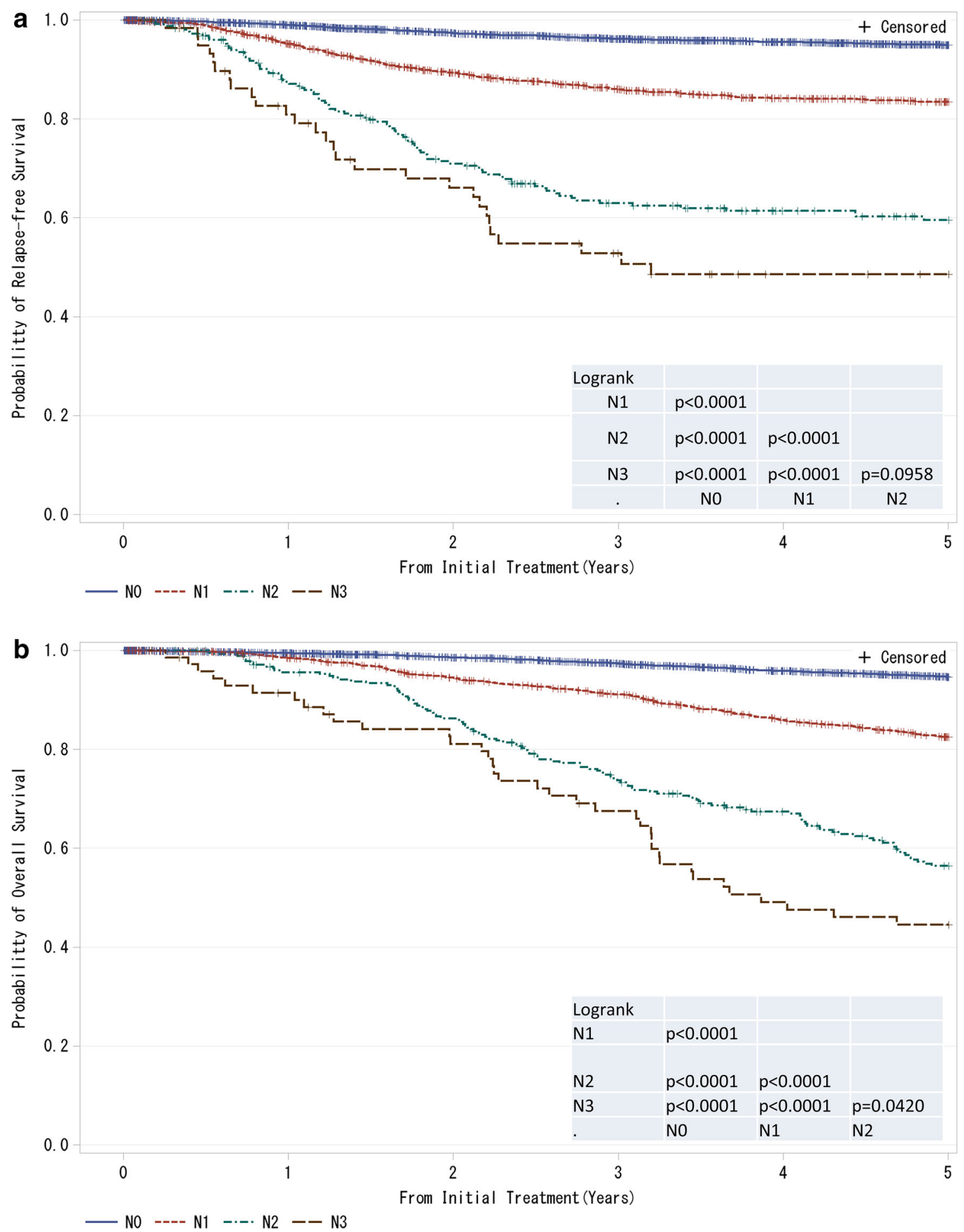

Fig. 2 a, b Kaplan-Meier curves for relapse-free and overall survival of all cases by regional lymph nodes status ( $\mathrm{cN}$-category) NO: No regional lymph node metastases; N1: Metastases in movable ipsilateral level I, II axillary lymph node(s); N2: Metastases in ipsilateral level I, II axillary lymph nodes that are clinically fixed or matted OR Metastases in clinically detected ipsilateral internal mammary nodes in the absence of clinically evident axillary lymph node metastases;
N3: Metastases in ipsilateral infraclavicular (level III axillary) lymph node(s) with or without level I, II axillary lymph node involvement OR Metastases in clinically detected ipsilateral internal mammary lymph node(s) with clinically evident level I, II axillary lymph node metastases OR Metastases in ipsilateral supraclavicular lymph node(s) with or without axillary or internal mammary lymph node involvement. $P$ values were calculated using the log-rank test 

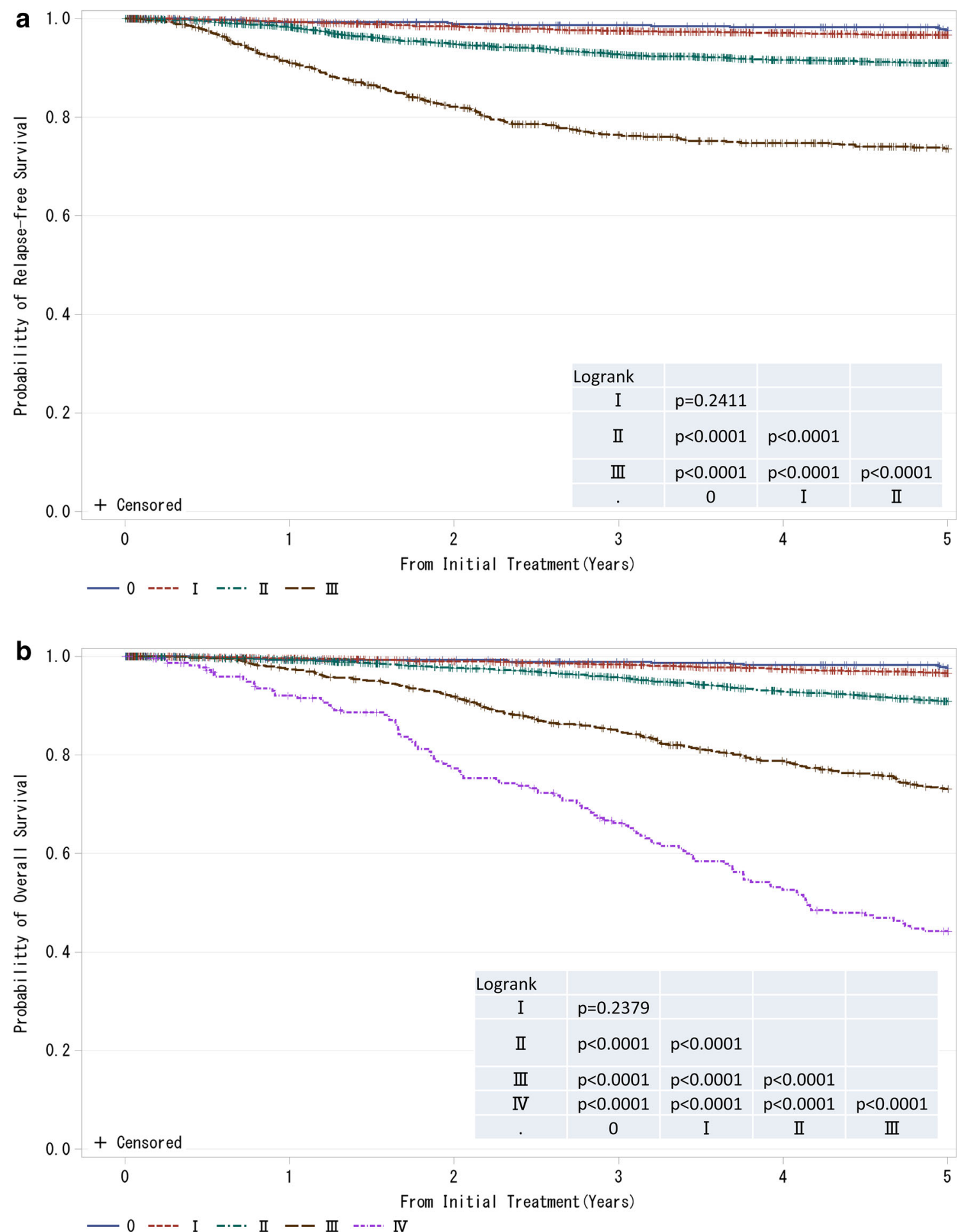

Fig. 3 a, b Kaplan-Meier curves for relapse-free and overall survival of all cases by clinical stage (UICC). $P$ values were calculated using the log-rank test 

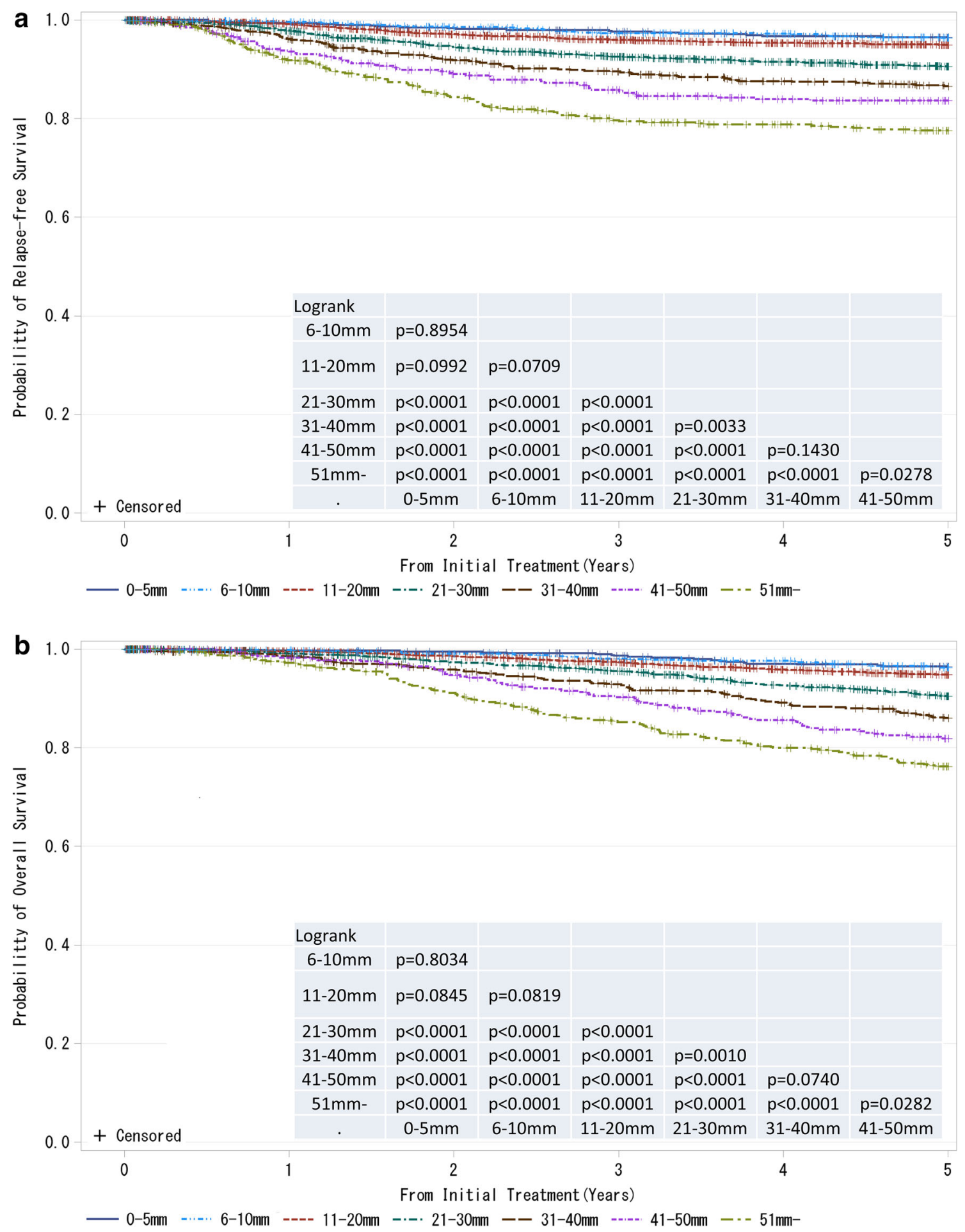

Fig. 4 a, b Kaplan-Meier curves for Relapse-free and overall survival of cases without neoadjuvant therapy by pathological tumor size (pT size). Tumor size is a marker of invasiveness. $P$ values were calculated using the log-rank test 

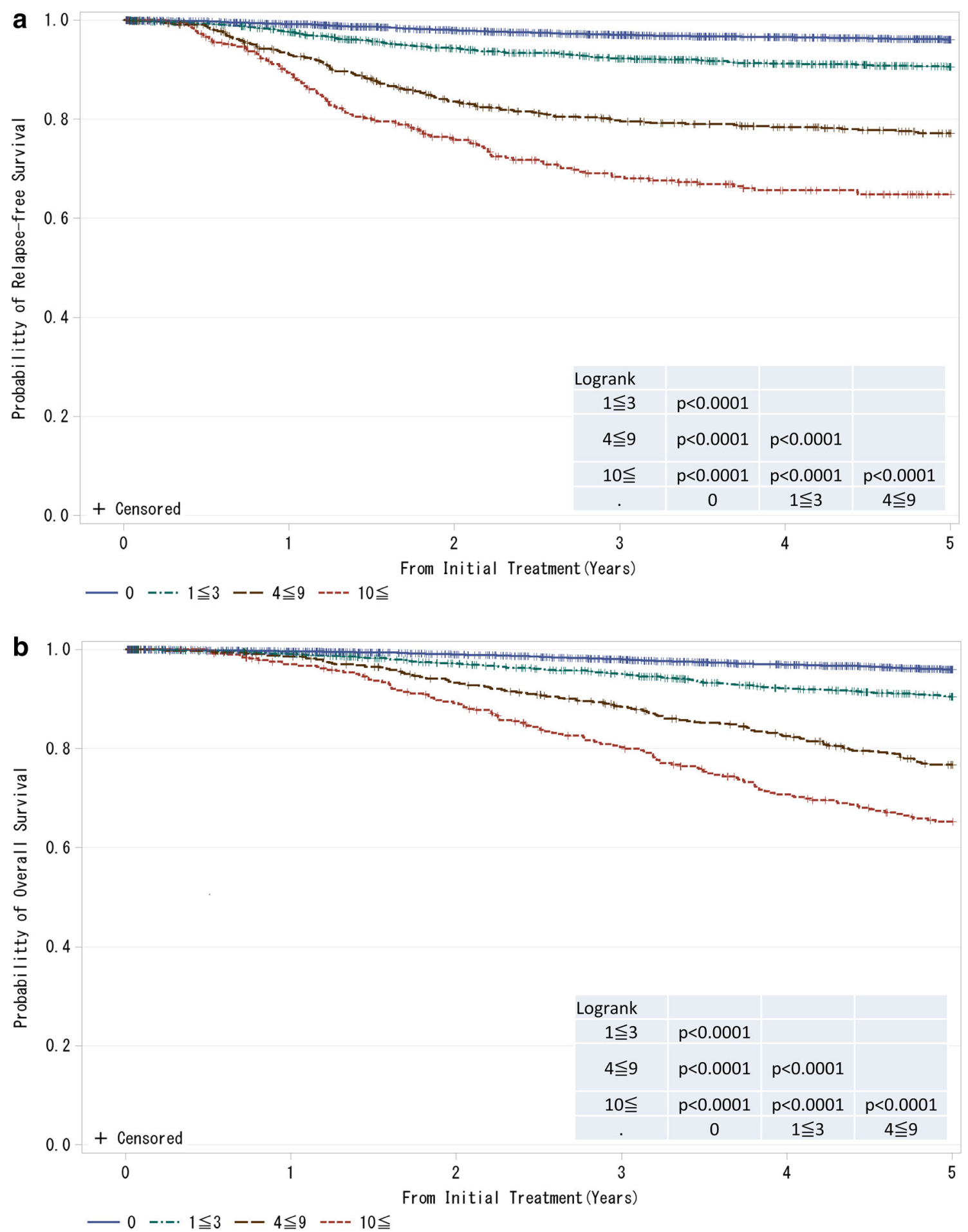

Fig. 5 a, b Kaplan-Meier curves for relapse-free and overall survival of cases without neoadjuvant therapy by the number of metastatic lymph nodes. $P$ values were calculated using the log-rank test 

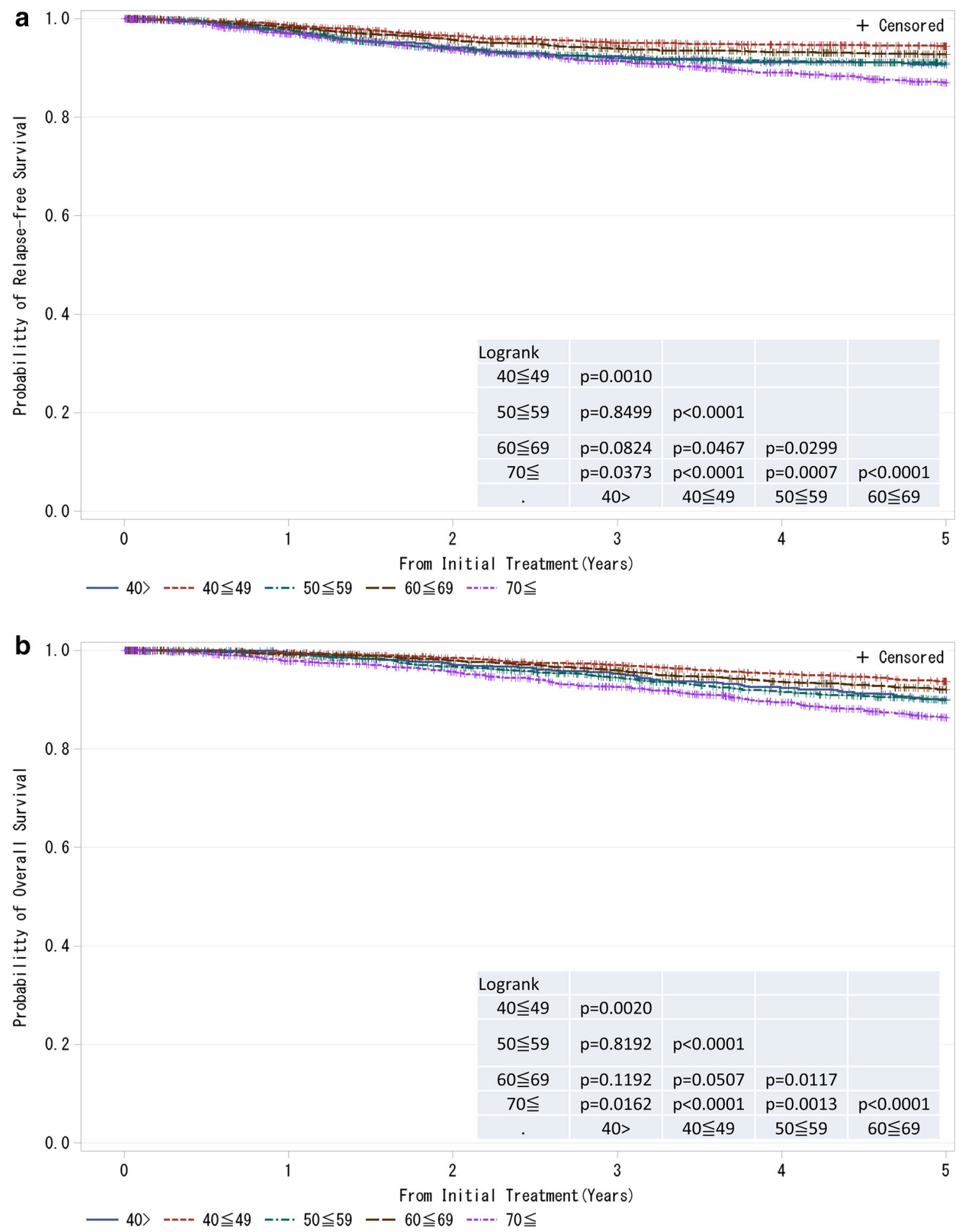

Fig. 6 a, b Kaplan-Meier curves for relapse-free and overall survival of all cases by age. $P$ values were calculated using the log-rank test

Background characteristics of the patients are summarized in Table 1. The 5-year Disease Free Survival (DFS) was $91.5 \%$, and the 5-year overall survival (OS) was $90.7 \%$ at a median follow-up of 60.0 months (range 0.0-60.0). TNM and histopathological classifications were determined using the UICC staging and WHO classification systems, respectively. TNM classification was performed according to the sixth edition of the American Joint Committee on Cancer (AJCC) staging system [1]. Histological classification was based on the WHO classification 

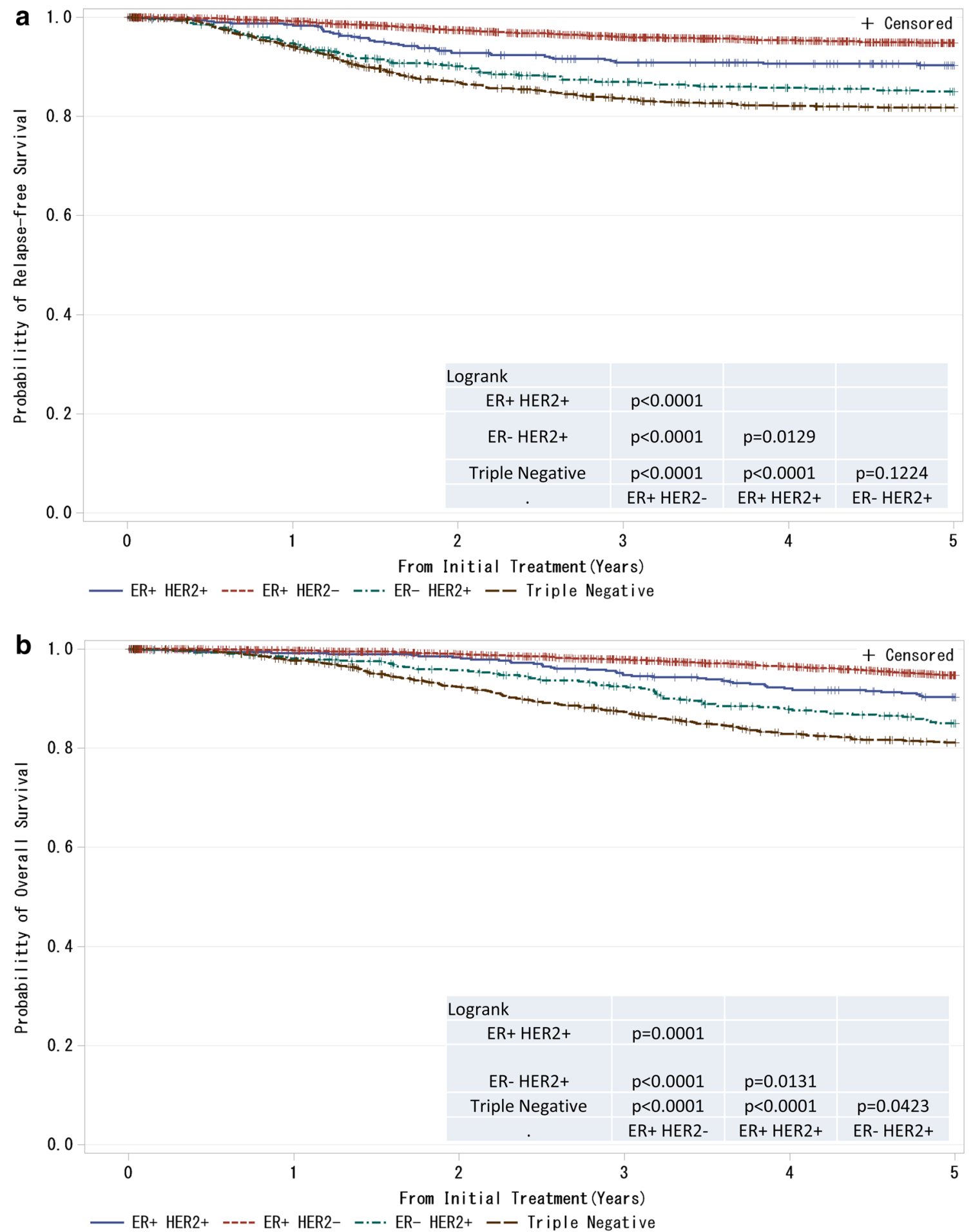

Fig. 7 a, b Kaplan-Meier curves for relapse-free and overall survival of T1-T4, any N and M0 cases with respect to estrogen receptor (ER) status and HER2 (human epidermal growth factor receptor 2)

system [2]. The present report includes age- and subtypebased analyses in addition to the traditional TNM classification-based analyses. In the clinical setting, Estrogen receptor (ER), Progesterone receptor (PgR), and HER2 amplification status. $P$ values were calculated using the log-rank test. Relapse-free survival and overall survival of patients with respect to combined ER and HER2 status

status, which are strong prognostic factors, have become frequently used to determine the therapeutic strategy. This trend has important implications for breast cancer treatment; therefore, we propose that subtype-based 

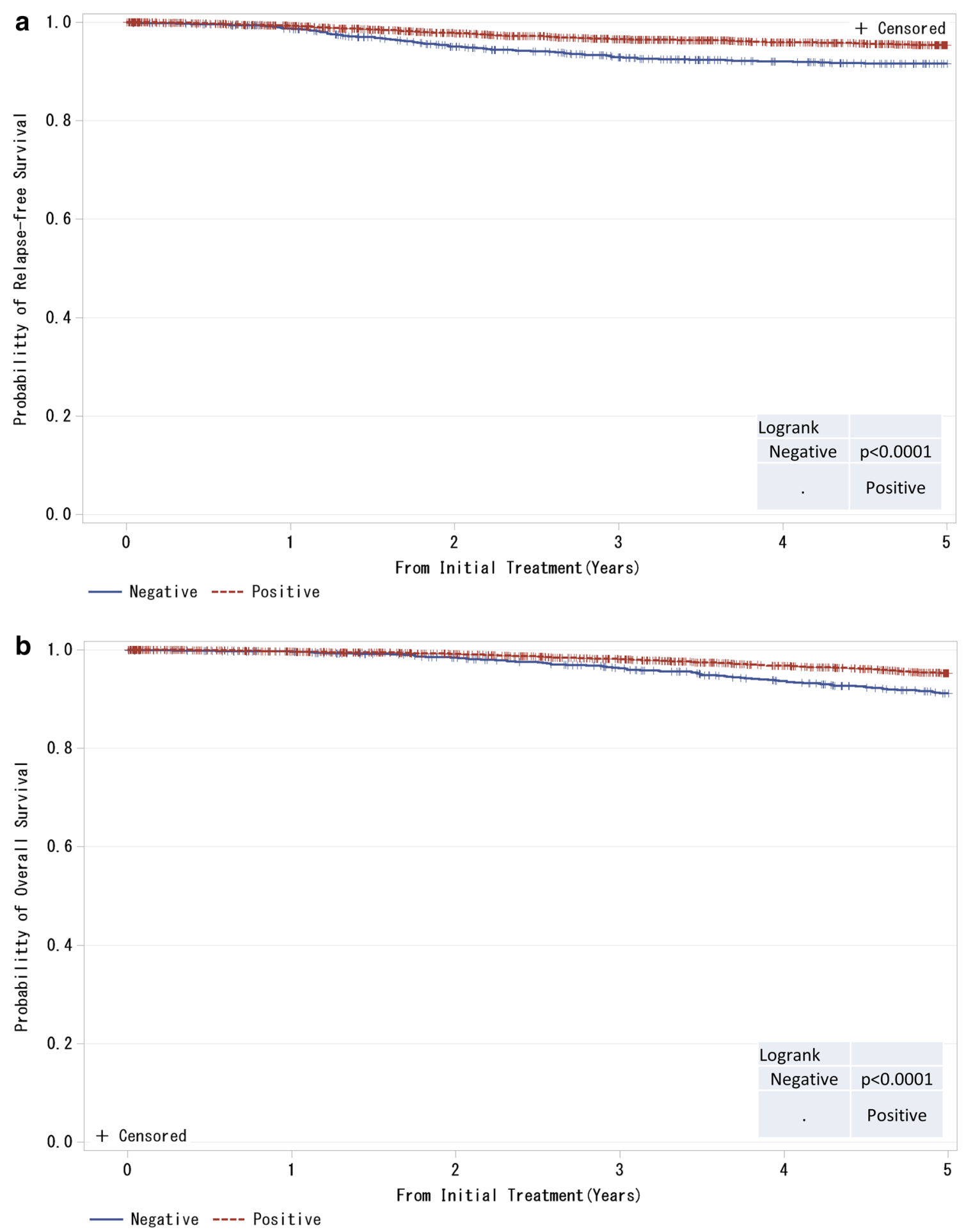

Fig. 8 a, b Kaplan-Meier curves for relapse-free and overall survival of ER-positive and M0 cases by progesterone receptor (PgR) status. $P$ values were calculated using the log-rank test

classification should be considered when the UICC's TNM classification system is next revised. Note that during the study period, trastuzumab was rarely used because it was not covered by the Japanese National Health Insurance program as adjuvant therapy for HER2-positive breast cancer. Recently, trastuzumab has been more frequently used to treat recurrent breast cancer. Thus, we believe that data in the registry such as the relapse-free survival of 


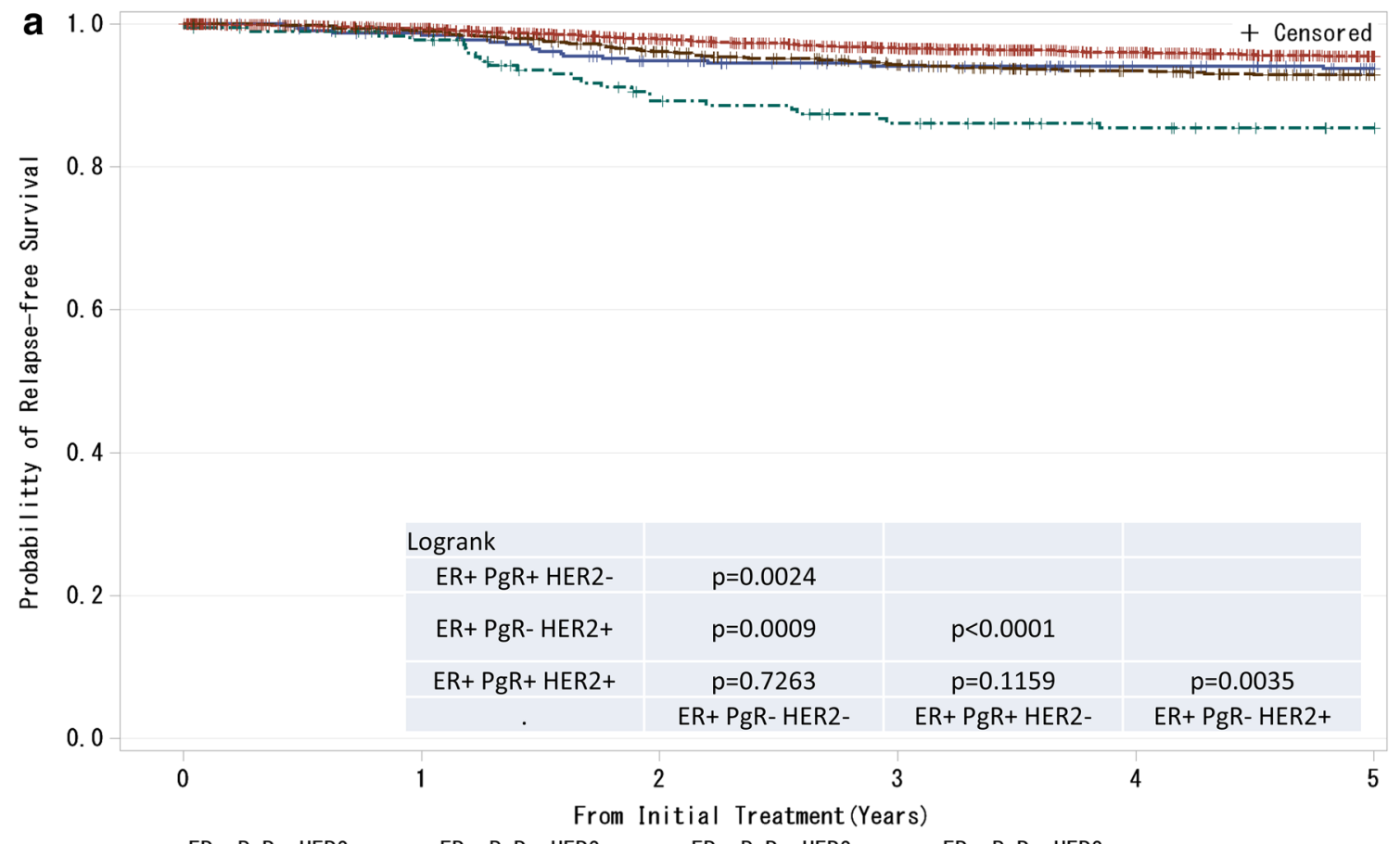

- ER+ PgR+ HER2+ ---- ER+ PgR+ HER2- ---. ER+ PgR- HER2+ - - ER+ PgR- HER2-
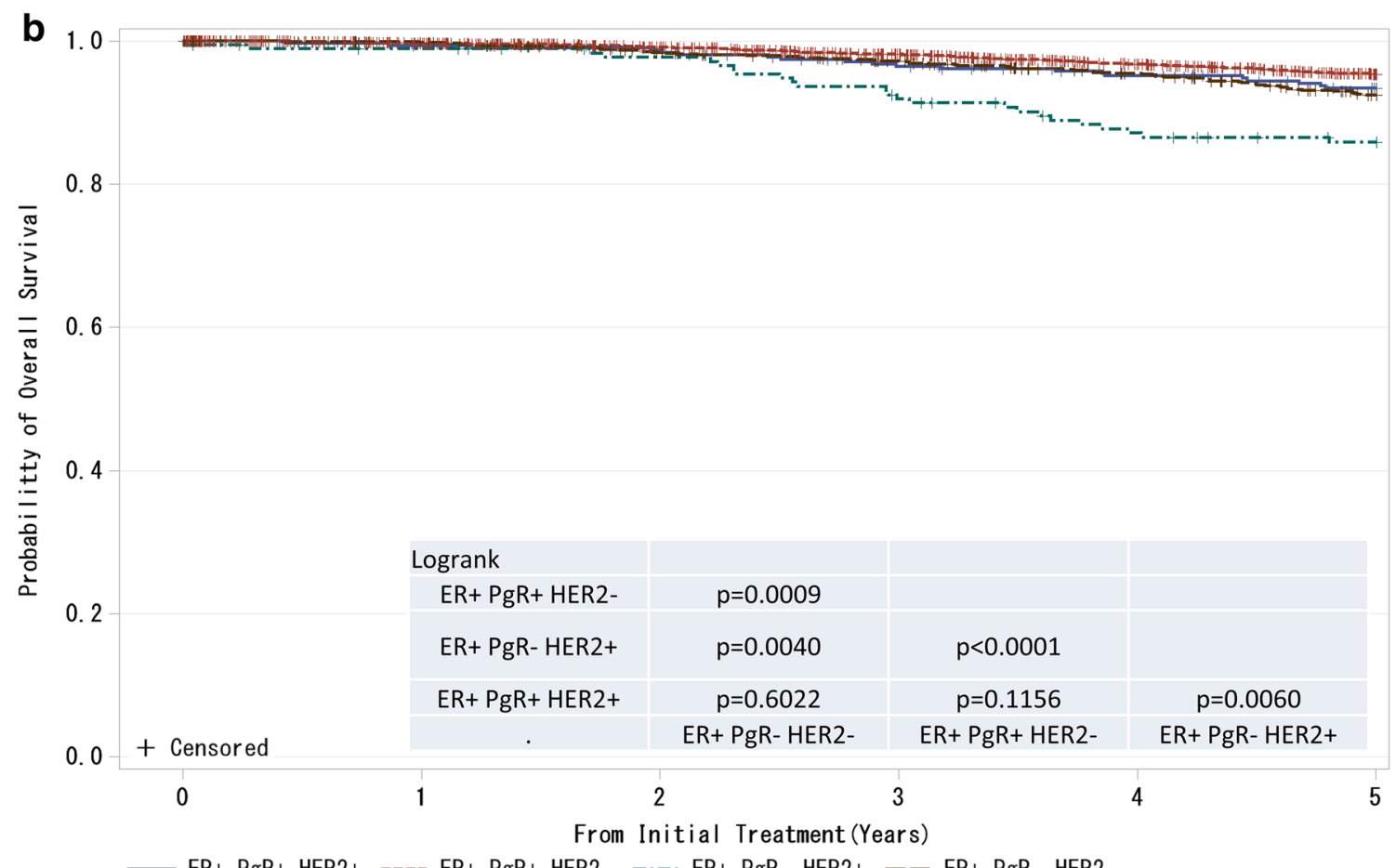

ER+ PgR+ HER2+ ---- ER+ PgR+ HER2- -.-. ER+ PgR- HER2+ -- ER+ PgR- HER2-

Fig. 9 a, b Kaplan-Meier curves for relapse-free and overall survival of ER-positive and M0 cases with respect to PgR and HER2 amplifications. $P$ values were calculated using the log-rank test

patients with HER2-positive breast cancer can be an important resource for comparison with data obtained after the drug has been covered by the insurance.
On January 1, 2012, the Breast Cancer Registry was incorporated in the current National Clinical Database (NCD) registration system. We appreciate the considerable 
Table 1 Patient characteristics

\begin{tabular}{|c|c|c|}
\hline Age & 56.99 (Mean) & 12.89 (S.D.) \\
\hline Tumor size $(\mathrm{cm})$ & 2.72 (Mean) & 2.08 (S.D.) \\
\hline \multicolumn{3}{|l|}{ Tumor size } \\
\hline T0 & 139 & 1.62 \\
\hline Tis & 592 & 6.9 \\
\hline T1a & 52 & 0.61 \\
\hline $\mathrm{T} 1 \mathrm{~b}$ & 655 & 7.63 \\
\hline $\mathrm{T} 1 \mathrm{c}$ & 2462 & 28.68 \\
\hline $\mathrm{T} 2$ & 3249 & 37.85 \\
\hline $\mathrm{T} 3$ & 396 & 4.61 \\
\hline $\mathrm{T} 4$ & 520 & 6.06 \\
\hline Unknown & 520 & 6.06 \\
\hline \multicolumn{3}{|l|}{$\mathrm{N}$} \\
\hline No & 6538 & 76.16 \\
\hline N1 & 1585 & 18.46 \\
\hline $\mathrm{N} 2$ & 277 & 3.23 \\
\hline N3 & 72 & 0.84 \\
\hline Unknown & 113 & 1.32 \\
\hline \multicolumn{3}{|l|}{ M } \\
\hline M0 & 8154 & 94.98 \\
\hline M1 & 224 & 2.61 \\
\hline Unknown & 207 & 2.41 \\
\hline \multicolumn{3}{|l|}{ Stage } \\
\hline 0 & 551 & 6.42 \\
\hline I & 2792 & 32.52 \\
\hline II & 3466 & 40.37 \\
\hline III & 725 & 8.44 \\
\hline IV & 224 & 2.61 \\
\hline Unknown & 827 & 9.63 \\
\hline \multicolumn{3}{|l|}{ ER } \\
\hline Positive & 6014 & 70.05 \\
\hline Negative & 2141 & 24.94 \\
\hline Unknown & 430 & 5.01 \\
\hline \multicolumn{3}{|l|}{$\operatorname{PgR}$} \\
\hline Positive & 4887 & 56.92 \\
\hline Negative & 3227 & 37.59 \\
\hline Unknown & 471 & 5.49 \\
\hline \multicolumn{3}{|l|}{ HER2 } \\
\hline Positive & 1213 & 14.13 \\
\hline Negative & 5879 & 68.48 \\
\hline Unknown & 1493 & 17.39 \\
\hline
\end{tabular}

The TNM classification was identified by the UICC staging system; $E R$ estrogen receptor, $P g R$ progesterone receptor, HER2 human epidermal growth factor receptor 2 support that we have received and would like to ask for continuing understanding and support of the registry.

Acknowledgments This work was supported partly by JSPS KAKENHI Grant Numbers 15H04796. We thank Dr. Muneaki Sano, a former director of Niigata Cancer Center Hospital, for his dedication to establishing the new registration system, and the staff members at the Japan Clinical Research Support Unit (J-CRSU) and the Public Health Research Foundation. We extend our gratitude to staff members working at the three major institutions that contributed extensively to the present study: Sagara Hospital (Kagoshima), Niigata Cancer Center Hospital (Niigata), and National Cancer Center Hospital (Tokyo). We would like to ask for support from as many institutions as possible in the future in order to improve response rates in our survey on breast cancer prognosis.

Open Access This article is distributed under the terms of the Creative Commons Attribution 4.0 International License (http://crea tivecommons.org/licenses/by/4.0/), which permits unrestricted use, distribution, and reproduction in any medium, provided you give appropriate credit to the original author(s) and the source, provide a link to the Creative Commons license, and indicate if changes were made.

\section{References}

1. World Health Organization. Tumours of the breast and female genital organs. Oxford: Oxford University Press; 2003. p. 2003.

2. Sobin L, Gosporarowicz M, Wittekind C. International union against cancer (UICC): TMM classification of malignant tumours. 7th ed. New York: Wiley; 2009. 